\title{
Synthetic approach to hypoxyxylerone, novel inhibitor of topoisomerase I
}

Arnaud Piettre, Emmanuel Chevenier, Christine Massardier, Yves Gimbert*, and Andrew E. Greene*

Université Joseph Fourier de Grenoble, Chimie Recherche (LEDSS), 38041 Grenoble Cedex, France

Yves.Gimbert(Andrew.Greene)@ujf-grenoble.fr

\section{SUPPORTING INFORMATION}

3-Benzyloxy-1,6,8-trimethoxy-naphthalene-2-carboxylic Acid 3-Bromo-4,5,7trimethoxy-naphthalen-2-yl Methyl Ester (4).

To a stirred solution of acid 5 (203 mg, $0.552 \mathrm{mmol})$, alcohol 6 (180 mg, $0.549 \mathrm{mmol})$, and triphenylphosphine $(159 \mathrm{mg}, 0.606 \mathrm{mmol})$ in anhydrous $\mathrm{THF}(6.8 \mathrm{~mL})$ at $0{ }^{\circ} \mathrm{C}$ under argon was added dropwise diethyl azodicarboxylate $(96 \mu \mathrm{L}, 0.610 \mathrm{mmol})$. Stirring was continued at room temperature for $3 \mathrm{~h}$, whereupon the mixture was diluted with ether and, after the usual workup, the crude product was chromatographed (silica gel, hexane-EtOAc 7:3). The residue obtained was dissolved in ether and washed several times with aqueous $\mathrm{HCl}$ $(6 \mathrm{~N})$ (to remove the byproduct of reduced DEAD) and brine, dried over anhydrous $\mathrm{Na}_{2} \mathrm{SO}_{4}$, filtered, and concentrated under reduced pressure to afford ester $\mathbf{4}$ as a white solid (356 mg, $95 \%)$.

mp $136-138{ }^{\circ} \mathrm{C}$ (hexane-EtOAc);

IR (neat) 2926, 2850, 1728, 1619, $1580 \mathrm{~cm}^{-1}$;

${ }^{1} \mathrm{H}$ NMR $\left(\mathrm{CDCl}_{3}, 200 \mathrm{MHz}\right) \delta 7.66(\mathrm{~s}, 1 \mathrm{H}), 7.40-7.10(\mathrm{~m}, 5 \mathrm{H}), 6.86(\mathrm{~s}, 1 \mathrm{H}), 6.59$ (d, $J=2.1$ $\mathrm{Hz}, 2 \mathrm{H}), 6.50(\mathrm{~d}, J=2.4 \mathrm{~Hz}, 1 \mathrm{H}), 6.39$ (d, $J=2.1 \mathrm{~Hz}, 1 \mathrm{H}), 5.58(\mathrm{~s}, 2 \mathrm{H}), 5.20$ (s, 2H), 3.94 (s, $3 \mathrm{H}), 3.93$ (s, 3H), 3.88 (s, 3H), 3.85 (s, 3H), 3.84 (s, 3H), 3.77 (s, 3H);

${ }^{13} \mathrm{C} \mathrm{NMR}\left(\mathrm{CDCl}_{3}, 50.3 \mathrm{MHz}\right) \delta 166.1(\mathrm{C}), 159.3(\mathrm{C}), 158.4(\mathrm{C}), 157.5$ (C), $156.4(\mathrm{C}), 155.4$ (C), $153.8(\mathrm{C}), 153.4(\mathrm{C}), 139.1(\mathrm{C}), 136.5$ (C), $134.1(\mathrm{C}), 128.4(\mathrm{CH}), 126.6(\mathrm{CH}), 123.2$ $(\mathrm{CH}), 117.1(\mathrm{C}), 116.2(\mathrm{C}), 113.0(\mathrm{C}), 110.7(\mathrm{C}), 103.5(\mathrm{CH}), 99.6(\mathrm{CH}), 98.9(\mathrm{CH}), 98.4$ 
$(\mathrm{CH}), 97.3(\mathrm{CH}), 69.9\left(\mathrm{CH}_{2}\right), 66.7\left(\mathrm{CH}_{2}\right), 63.9\left(\mathrm{CH}_{3}\right), 61.5\left(\mathrm{CH}_{3}\right), 56.1\left(\mathrm{CH}_{3}\right), 55.9\left(\mathrm{CH}_{3}\right)$, $55.2\left(\mathrm{CH}_{3}\right), 55.1\left(\mathrm{CH}_{3}\right)$;

MS (DCI) m/z $679\left(\mathrm{MH}^{+}, 100\right), 369$ (8), 351 (41), 311 (77), 233 (14);

HRMS calcd for $\mathrm{C}_{35} \mathrm{H}_{34} \mathrm{BrO}_{9}$ : 677.1386. Found: $677.1339\left(\mathrm{MH}^{+}\right)$.

\section{1-(3-Benzyloxy-1,6,8-trimethoxy-naphthalen-2-yl)-1-(3-hydroxymethyl-1,6,8-} trimethoxy-naphthalen-2-yl)-methanone (20).

Under argon, ester 4 (269 mg, $0.397 \mathrm{mmol})$ was dissolved in THF $(1.59 \mathrm{~mL}, \mathrm{C}=0.25$ $\mathrm{M}$, from which residual water had been removed with $n$-butyllithium and $o$-phenanthroline), and cooled to $-45 /-55{ }^{\circ} \mathrm{C}$. $n$-Butyllithium $(203 \mu \mathrm{L}, 0.437 \mathrm{mmol})$ was then added dropwise. After the solution was stirred for $2 \mathrm{~h}$, the reaction mixture was quenched by addition of saturated aqueous $\mathrm{NH}_{4} \mathrm{Cl}$, allowed to warm to ambient temperature, and diluted with ethyl acetate. After the usual workup, the crude product was purified by radial thin-layer chromatography (hexane-EtOAc 9:1, 8:2, 7:3, and then 5:5) to give a white solid 20 (130 mg, $55 \%)$.

$\mathrm{mp} 220-224^{\circ} \mathrm{C}$ (cyclohexane-dichloromethane);

IR (neat) 3454, 2957, 2926, 2853, 1619, 1573, $1458 \mathrm{~cm}^{-1}$;

${ }^{1} \mathrm{H}$ NMR $\left(\mathrm{CDCl}_{3}, 200 \mathrm{MHz}\right) \delta 7.41(\mathrm{~s}, 1 \mathrm{H}), 7.35-7.05(\mathrm{~m}, 5 \mathrm{H}), 6.87(\mathrm{~s}, 1 \mathrm{H}), 6.70(\mathrm{~d}, J=2.1$ $\mathrm{Hz}, 1 \mathrm{H}), 6.60(\mathrm{~d}, J=2.1 \mathrm{~Hz}, 1 \mathrm{H}), 6.45$ (d, $J=2.4 \mathrm{~Hz}, 1 \mathrm{H}), 6.37$ (d, $J=2.4 \mathrm{~Hz}, 1 \mathrm{H}), 5.03$ (s, $2 \mathrm{H}), 4.55(\mathrm{~d}, J=6.8 \mathrm{~Hz}, 2 \mathrm{H}), 3.90(\mathrm{~s}, 6 \mathrm{H}), 3.87(\mathrm{~s}, 3 \mathrm{H}), 3.83(\mathrm{~s}, 3 \mathrm{H}), 3.57(\mathrm{~s}, 3 \mathrm{H}), 3.41$ (s, $3 \mathrm{H})$;

${ }^{13} \mathrm{C} \mathrm{NMR}\left(\mathrm{CDCl}_{3}, 75.4 \mathrm{MHz}\right) \delta 198.6(\mathrm{C}), 159.6(\mathrm{C}), 159.4$ (C), 157.8 (C), 157.6 (C) 156.0 (C), 155.1 (C), 139.5 (C), 139.3 (C), 136.3 (C), 131.3 (C), $128.3(\mathrm{CH}), 127.8$ (CH), 127.4 (CH), $125.4(\mathrm{C}), 124.4(\mathrm{CH}), 114.7(\mathrm{C}), 111.1(\mathrm{C}), 103.2(\mathrm{CH}), 99.3(\mathrm{CH}), 98.7(\mathrm{CH}), 97.4$ $(\mathrm{CH}), 70.2\left(\mathrm{CH}_{2}\right), 64.7\left(\mathrm{CH}_{2}\right), 64.1\left(\mathrm{CH}_{3}\right), 64.0\left(\mathrm{CH}_{3}\right), 63.9\left(\mathrm{CH}_{3}\right), 56.0\left(\mathrm{CH}_{3}\right), 55.4\left(\mathrm{CH}_{3}\right)$, $55.3\left(\mathrm{CH}_{3}\right)$;

MS (DCI, $\mathrm{NH}_{3}+$ isobutane) m/z $599\left(\mathrm{MH}^{+}, 50\right), 279$ (100);

HRMS calcd for $\mathrm{C}_{35} \mathrm{H}_{34} \mathrm{O}_{9}$ : 598.2203. Found: $598.2211\left(\mathrm{MH}^{+}\right)$. 


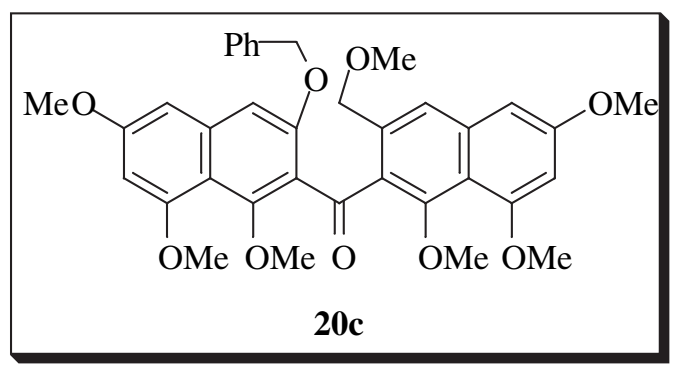

To a solution of alcohol $20(180 \mathrm{mg}, 0.301 \mathrm{mmol})$ in dichloromethane $(3.2 \mathrm{~mL})$ at room temperature was added silver (I) oxide $(280 \mathrm{mg}, 1.25 \mathrm{mmol})$ and iodomethane $(150 \mu \mathrm{L}$, $2.41 \mathrm{mmol}$ ). The reaction mixture was stirred in the dark. After 48 h, 72 h, 96 h, and 144 h, additional silver (I) oxide (145 mg, $0.626 \mathrm{mmol})$ and iodomethane (70 $\mu \mathrm{L}, 1.12 \mathrm{mmol})$ were added (until disappearance of the starting material in thin-layer chromatography). The slurry was then filtered and the solvent was removed in vacuo to give the product 20c $(190 \mathrm{mg})$.

mp $175-178{ }^{\circ} \mathrm{C}$ (pentane-dichloromethane, white solid);

IR (neat) 2935, 2845, 1662, 1620, $1578 \mathrm{~cm}^{-1}$;

${ }^{1} \mathrm{H}$ NMR $\left(\mathrm{CDCl}_{3}, 200 \mathrm{MHz}\right) \delta 7.65(\mathrm{~s}, 1 \mathrm{H}), 7.20(\mathrm{~s}, 5 \mathrm{H}), 6.86(\mathrm{~s}, 1 \mathrm{H}), 6.74(\mathrm{~d}, J=2.1 \mathrm{~Hz}$, $1 \mathrm{H}), 6.59(\mathrm{~d}, J=2.1 \mathrm{~Hz}, 1 \mathrm{H}), 6.43(\mathrm{~d}, J=2.1 \mathrm{~Hz}, 1 \mathrm{H}), 6.35(\mathrm{~d}, J=2.4 \mathrm{~Hz}, 1 \mathrm{H}), 5.08(\mathrm{~s}, 2 \mathrm{H})$, 4.73 (s, 2H), 3.88 (s, 6H), 3.85 (s, 3H), 3.83 (s, 3H), 3.47 (s, 3H), 3.43 (s, 3H);

${ }^{13} \mathrm{C}$ NMR $\left(\mathrm{CDCl}_{3}, 75.4 \mathrm{MHz}\right) \delta 196.6(\mathrm{C}), 159.2(2 \mathrm{C}), 157.7$ (C), $157.6(\mathrm{C}), 156.6(\mathrm{C}), 156.0$ (C), $155.1(\mathrm{C}), 139.2(\mathrm{C}), 138.9$ (C), 137.1 (C), $136.4(\mathrm{C}), 130.2(\mathrm{C}), 128.3(\mathrm{CH}), 127.6(\mathrm{CH})$, $127.1(\mathrm{CH}), 125.3(\mathrm{C}), 120.7(\mathrm{CH}), 114.0(\mathrm{C}), 110.8(\mathrm{C}), 103.3(\mathrm{CH}), 99.1(\mathrm{CH}), 98.7(\mathrm{CH})$, $98.5(\mathrm{CH}), 97.1(\mathrm{CH}), 71.8\left(\mathrm{CH}_{2}\right), 70.0\left(\mathrm{CH}_{2}\right), 64.0\left(\mathrm{CH}_{3}\right), 63.6\left(\mathrm{CH}_{3}\right), 58.6\left(\mathrm{CH}_{3}\right), 55.9$ $\left(2 \mathrm{CH}_{3}\right), 55.3\left(\mathrm{CH}_{3}\right), 55.2\left(\mathrm{CH}_{3}\right)$;

MS (EI) m/z $612\left(\mathrm{M}^{+}, 43\right), 581$ (100), 459 (41);

HRMS calcd for $\mathrm{C}_{36} \mathrm{H}_{36} \mathrm{LiO}_{9}$ : 619.2519. Found: $619.2536\left(\mathrm{MLi}^{+}\right)$. 


\section{1-(3-Hydroxy-1,6,8-trimethoxy-naphthalen-2-yl)-1-(1,6,8-trimethoxy-3-}

methoxymethyl-naphthalen-2-yl)-methanone (20d).

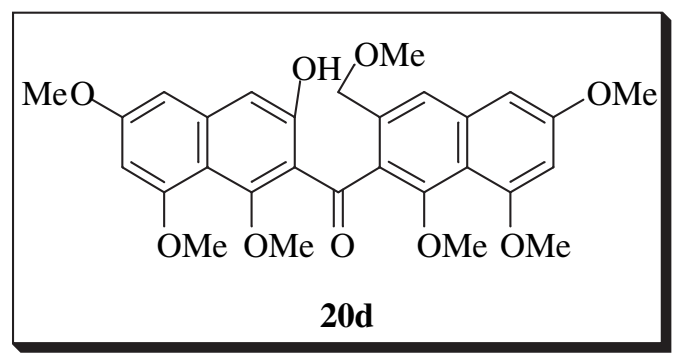

The product 20c $(190 \mathrm{mg})$ and $\mathrm{Pd}(\mathrm{OH})_{2} / \mathrm{C}(68 \mathrm{mg})$ were stirred in dichloromethane $(5.2 \mathrm{~mL})$ and ethanol $(13 \mathrm{~mL})$ under a hydrogen atmosphere for $16 \mathrm{~h}$. After removal of the catalyst by filtration, the filtrate was concentrated under reduced pressure to give a yellow solid (170 mg).

$\operatorname{mp} 115-116^{\circ} \mathrm{C}$;

IR (KBr) 3452, 2935, 2849, 1640, 1621, $1560 \mathrm{~cm}^{-1}$;

${ }^{1} \mathrm{H}$ NMR $\left(\mathrm{CDCl}_{3}, 200 \mathrm{MHz}\right) \delta 11.97(\mathrm{~s}, 1 \mathrm{H}), 7.47(\mathrm{~s}, 1 \mathrm{H}), 6.92(\mathrm{~s}, 1 \mathrm{H}), 6.74(\mathrm{~d}, J=2.1 \mathrm{~Hz}$, $1 \mathrm{H}), 6.52(\mathrm{~d}, J=2.1 \mathrm{~Hz}, 1 \mathrm{H}), 6.49(\mathrm{~d}, J=2.4 \mathrm{~Hz}, 1 \mathrm{H}), 6.19(\mathrm{~d}, J=2.1 \mathrm{~Hz}, 1 \mathrm{H}), 4.41(\mathrm{~s}, 2 \mathrm{H})$, $3.92(\mathrm{~s}, 3 \mathrm{H}), 3.90$ (s, 3H), 3.87 (s, 3H), 3.78 (s, 3H), 3.76 (s, 3H), 3.24 (s, 3H), 3.17 (s, 3H);

${ }^{13} \mathrm{C}$ NMR $\left(\mathrm{CDCl}_{3}, 75.4 \mathrm{MHz}\right) \delta 201.5(\mathrm{C}), 162.8(2 \mathrm{C}), 161.3(\mathrm{C}), 158.8(\mathrm{C}), 158.6(\mathrm{C}), 158.3$ (C), $157.4(\mathrm{C}), 152.9$ (C), 142.6 (C), 137.6 (C), 134.5 (C), 132.1 (C), $121.4(\mathrm{CH}), 115.9$ (C), $114.8(\mathrm{C}), 110.1(\mathrm{C}), 107.1(\mathrm{CH}), 99.1(2 \mathrm{CH}), 97.4(\mathrm{CH}), 96.7(\mathrm{CH}), 72.4\left(\mathrm{CH}_{2}\right), 64.2\left(\mathrm{CH}_{3}\right)$, $63.4\left(\mathrm{CH}_{3}\right), 58.4\left(\mathrm{CH}_{3}\right), 56.0\left(\mathrm{CH}_{3}\right), 55.7\left(\mathrm{CH}_{3}\right), 55.3\left(\mathrm{CH}_{3}\right), 55.2\left(\mathrm{CH}_{3}\right)$; MS (DCI, $\mathrm{NH}_{3}+$ isobutane) m/z 523( $\left.\mathrm{MH}^{+}, 100\right), 493$ (19), 289 (15), 261 (64), 233 (22); HRMS calcd for $\mathrm{C}_{29} \mathrm{H}_{30} \mathrm{LiO}_{9}$ : 529.2050. Found: $529.2071\left(\mathrm{MLi}^{+}\right)$.

\section{1,3,8,9,11-Pentamethoxy-6-methoxymethyl-14-oxa-benzo[ $\alpha]$ naphthacen-7-one (2).}

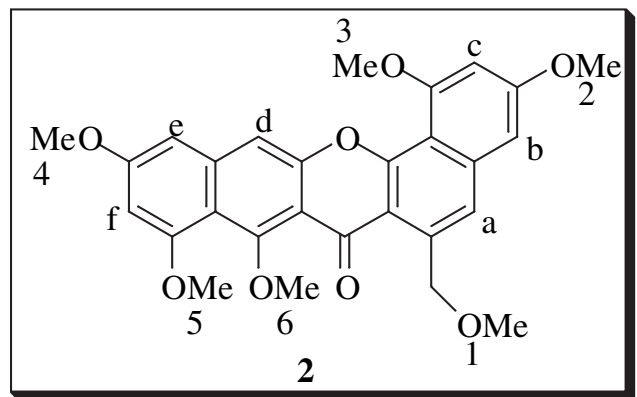


A solution of the product $\mathbf{2 0 d}(170 \mathrm{mg})$ and potassium hydroxide $(46 \mathrm{mg}, 0.829 \mathrm{mmol})$ in methanol $(10.9 \mathrm{~mL})$ was refluxed overnight. After being allowed to cool to room temperature, the reaction was quenched by addition of dilute aqueous $\mathrm{HCl}(0.1 \mathrm{~N})$ and the solvents were removed under reduced pressure. The residue was treated with aqueous $\mathrm{HCl}$ $(0.1 \mathrm{~N})$ and then extracted with dichloromethane. After the usual workup, the resultant crude product was triturated with ether to leave xanthone 2 as a yellow solid (155 mg, $91 \%$ overall yield for three steps).

$\operatorname{mp} 238-240{ }^{\circ} \mathrm{C}$;

IR (KBr) 2936, 2850, 1647, 1617, $1558 \mathrm{~cm}^{-1}$;

UV $\lambda \max \left(\mathrm{CHCl}_{3}\right) / \mathrm{nm} 242$ (log $\left.\varepsilon, 4.63\right), 297$ (4.83), 412 (4.07);

${ }^{1} \mathrm{H} \mathrm{NMR}\left(\mathrm{CDCl}_{3}, 500 \mathrm{MHz}\right) \delta 7.74\left(\mathrm{~s}, 1 \mathrm{H}, \mathrm{H}_{\mathrm{a}}\right), 7.48\left(\mathrm{~s}, 1 \mathrm{H}, \mathrm{H}_{\mathrm{d}}\right), 6.76\left(\mathrm{~d}, J=2.4 \mathrm{~Hz}, 1 \mathrm{H}, \mathrm{H}_{\mathrm{b}}\right)$, $6.67\left(\mathrm{~d}, J=2.1 \mathrm{~Hz}, 1 \mathrm{H}, \mathrm{H}_{\mathrm{e}}\right), 6.56\left(\mathrm{~d}, J=2.4 \mathrm{~Hz}, 1 \mathrm{H}, \mathrm{H}_{\mathrm{c}}\right), 6.40\left(\mathrm{~d}, J=2.1 \mathrm{~Hz}, 1 \mathrm{H}, \mathrm{H}_{\mathrm{f}}\right), 5.28(\mathrm{~s}$, 2H), $4.06\left(\mathrm{~s}, 3 \mathrm{H}, \mathrm{H}_{3}\right.$ or $\left.\mathrm{H}_{6}\right), 4.04\left(\mathrm{~s}, 3 \mathrm{H}, \mathrm{H}_{3}\right.$ or $\left.\mathrm{H}_{6}\right), 3.97\left(\mathrm{~s}, 3 \mathrm{H}, \mathrm{H}_{5}\right), 3.92\left(\mathrm{~s}, 3 \mathrm{H}, \mathrm{H}_{4}\right.$ ou $\left.\mathrm{H}_{2}\right)$, 3.91 (s, $3 \mathrm{H}, \mathrm{H}_{4}$ ou $\left.\mathrm{H}_{2}\right), 3.62$ (s, $\left.3 \mathrm{H}, \mathrm{H}_{1}\right)$;

${ }^{13} \mathrm{C} \mathrm{NMR}\left(\mathrm{CDCl}_{3}, 125.7 \mathrm{MHz}\right) \delta 178.2(\mathrm{C}), 161.1(\mathrm{C}), 160.6$ (C), $160.0(\mathrm{C}), 159.6$ (C), 159.4 (C), 155.6 (C), 153.4 (C), 140.3 (C), 139.8 (C), 138.1 (C), 119.1 (CH, Ca), 115.4 (C), 114.0 (2C), $109.5(\mathrm{C}), 108.3(\mathrm{CH}, \mathrm{Cd}), 99.6(\mathrm{CH}, \mathrm{Cb}$ or $\mathrm{Cc}), 99.3(\mathrm{CH}, \mathrm{Cb}$ or $\mathrm{Cc}), 98.5$ (CH, Cf), $97.3(\mathrm{CH}, \mathrm{Ce}), 73.8\left(\mathrm{CH}_{2}\right), 63.4\left(\mathrm{CH}_{3}, \mathrm{C} 3\right.$ or $\left.\mathrm{C} 6\right), 59.0\left(\mathrm{CH}_{3}, \mathrm{C} 1\right), 56.3\left(2 \mathrm{CH}_{3}, \mathrm{C} 5\right.$ and $\mathrm{C} 3$ or C6), $55.5\left(2 \mathrm{CH}_{3}, \mathrm{C} 2\right.$ and $\left.\mathrm{C} 4\right)$;

MS (EI) $490\left(\mathrm{M}^{+}, 5\right), 475$ (50), $232(20)$;

HRMS calcd for $\mathrm{C}_{28} \mathrm{H}_{27} \mathrm{O}_{8}$ : 491.1706. Found: $491.1705\left(\mathrm{MH}^{+}\right)$.

\section{8-Hydroxy-1,3,9,11-tetramethoxy-6-methoxymethyl-14-oxa-benzo[ $\alpha]$ naphthacen-} 7-one (21).

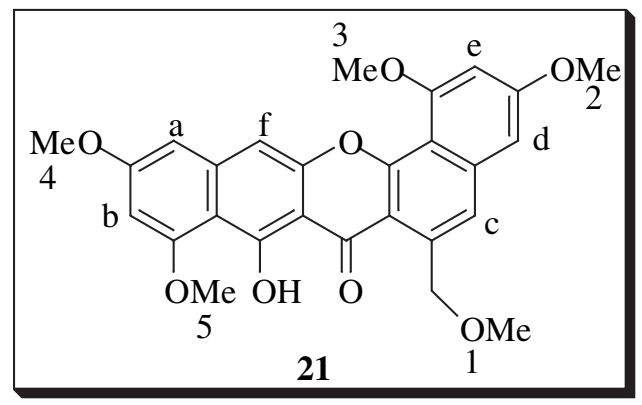


A solution of xanthone 2 (95 mg, $0.194 \mathrm{mmol})$ and lithium chloride (40 mg, 0.944 mmol) in DMF (2.8 mL) was stirred at $110{ }^{\circ} \mathrm{C}$ for $13 \mathrm{~h}$. After being allowed to cool to room temperature, the mixture was diluted with water and then extracted with chloroform. The organic layer was washed several times with water and then brine, dried over anhydrous $\mathrm{Na}_{2} \mathrm{SO}_{4}$, filtered, and concentrated in vacuo to give xanthone 21 as a red-brown solid (82 $\mathrm{mg}$, $89 \%)$.

mp $268-269^{\circ} \mathrm{C}$;

IR (KBr) 3411, 2925, 1650, 1620, 1602, $1573 \mathrm{~cm}^{-1}$;

${ }^{1} \mathrm{H} \mathrm{NMR}\left(\mathrm{CDCl}_{3}, 500 \mathrm{MHz}\right) \delta 7.74$ (s, 1H, Hc), 7.10 (s, 1H, Hf), 6.80 (d, J=2 Hz, 1H, Hd), $6.66(\mathrm{~d}, J=2.5 \mathrm{~Hz}, 1 \mathrm{H}, \mathrm{Ha}), 6.61(\mathrm{~d}, J=2 \mathrm{~Hz}, 1 \mathrm{H}, \mathrm{He}), 6.39$ (d, $J=2 \mathrm{~Hz}, 1 \mathrm{H}, \mathrm{Hb}), 5.26$ (d, $J=1 \mathrm{~Hz}, 2 \mathrm{H}), 4.10(\mathrm{~s}, 3 \mathrm{H}, \mathrm{C} 3), 4.03$ (s, 3H, C5), 3.95 (s, 3H, C4), 3.95 (s, 3H, C2), 3.66 (s, $3 \mathrm{H}, \mathrm{C} 1)$;

${ }^{13} \mathrm{C} \mathrm{NMR}\left(\mathrm{CDCl}_{3}, 75.4 \mathrm{MHz}\right) \delta 183.8(\mathrm{C}), 163.9(\mathrm{C}), 161.8(\mathrm{C}), 161.7$ (C), $161.0(\mathrm{C}), 159.8$ (C), 157.2 (C), 152.0 (C), 141.9 (C), 140.5 (C), 137.5 (C), 119.7 (CH), 112.4 (C), 109.7 (C), $108.2(\mathrm{C}), 104.4(\mathrm{C}), 100.8(\mathrm{CH}), 99.8(\mathrm{CH}), 99.6(\mathrm{CH}), 97.9(\mathrm{CH}), 96.9(\mathrm{CH}), 73.6\left(\mathrm{CH}_{2}\right)$, $59.1\left(\mathrm{CH}_{3}\right), 56.4\left(\mathrm{CH}_{3}\right), 56.3\left(\mathrm{CH}_{3}\right), 55.6\left(\mathrm{CH}_{3}\right), 55.5\left(\mathrm{CH}_{3}\right)$;

MS (DCI, $\mathrm{NH}_{3}+$ isobutane) m/z $477\left(\mathrm{MH}^{+}, 16\right), 102$ (100).

\section{1,3,9,11-Tetramethoxy-6-methoxymethyl-7H-14-oxa-benzo[ $\alpha]$ naphthacen-8-ol} (22).

To a solution of xanthone $21(37.5 \mathrm{mg}, 0.079 \mathrm{mmol})$ in dry dichloromethane $(7.9 \mathrm{~mL})$ was added dropwise borane-methyl sulfide complex $(75 \mu 1,0.791 \mathrm{mmol})$. The mixture was refluxed for $48 \mathrm{~h}$, and then allowed to cool to room temperature, diluted with an aqueous solution of $\mathrm{HCl}(0.5 \mathrm{~N})$, and extracted with dichloromethane. After the usual workup, xanthene 22 (37.1 mg) was obtained, which was used without further purification.

${ }^{1} \mathrm{H}$ NMR $\left(\mathrm{CDCl}_{3}, 300 \mathrm{MHz}\right) \delta 7.39(\mathrm{~s}, 1 \mathrm{H}), 6.94(\mathrm{~s}, 1 \mathrm{H}), 6.69(\mathrm{~d}, J=2.4 \mathrm{~Hz}, 1 \mathrm{H}), 6.63(\mathrm{~d}$, $J=2.1 \mathrm{~Hz}, 1 \mathrm{H}), 6.53(\mathrm{~d}, J=2.4 \mathrm{~Hz}, 1 \mathrm{H}), 6.32(\mathrm{~d}, J=2 \mathrm{~Hz}, 1 \mathrm{H}), 4.63(\mathrm{~s}, 2 \mathrm{H}), 4.07(\mathrm{~s}, 2 \mathrm{H})$, $4.02(\mathrm{~s}, 3 \mathrm{H}), 4.01(\mathrm{~s}, 3 \mathrm{H}), 3.88(\mathrm{~s}, 3 \mathrm{H}), 3.87(\mathrm{~s}, 3 \mathrm{H}), 3.48(\mathrm{~s}, 3 \mathrm{H})$;

MS (DCI, $\mathrm{NH}_{3}+$ isobutane) m/z 461 (18), 197 (100). 
Trimethyl-(1,3,6,9,11-tetramethoxy-6-methoxymethyl-7H-14-oxabenzo $[\alpha]$ naphthacen-8-yloxy)-silane (22c).

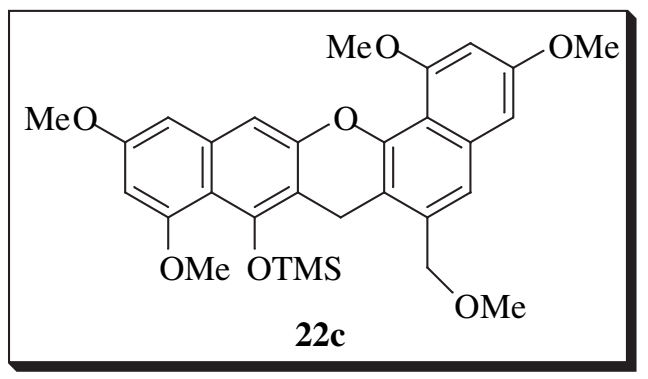

To a stirred solution of crude product $22(37.1 \mathrm{mg})$ in anhydrous dichloromethane $(0.85 \mathrm{~mL})$ at $0{ }^{\circ} \mathrm{C}$ were added dropwise 2,6 -lutidine $(64 \mu \mathrm{L}, 0.552 \mathrm{mmol})$ and then trimethylsilyl trifluoromethanesulfonate $(71 \mu \mathrm{L}, 0.394 \mathrm{mmol})$. After $2.5 \mathrm{~h}$, the reaction mixture was quenched by addition of saturated aqueous $\mathrm{NH}_{4} \mathrm{Cl}$ and then extracted with dichloromethane. The organic layer was washed with water, saturated aqueous $\mathrm{CuSO}_{4}$, and brine, dried over $\mathrm{Na}_{2} \mathrm{SO}_{4}$, filtered, and concentrated under reduced pressure to afford xanthene 22c (42.3 mg) as a green substance, which was used without further purification.

${ }^{1} \mathrm{H}$ NMR $\left(\mathrm{CDCl}_{3}, 300 \mathrm{MHz}\right) \delta 7.36(\mathrm{~s}, 1 \mathrm{H}), 7.10(\mathrm{~s}, 1 \mathrm{H}), 6.68(\mathrm{~d}, J=2.3 \mathrm{~Hz}, 1 \mathrm{H}), 6.62(\mathrm{~d}$, $J=2.3 \mathrm{~Hz}, 1 \mathrm{H}), 6.54(\mathrm{~d}, J=2.3 \mathrm{~Hz}, 1 \mathrm{H}), 6.32(\mathrm{~d}, J=2.3 \mathrm{~Hz}, 1 \mathrm{H}), 4.59$ (s, 2H), 4.09 (s, 2H), 4.03 (s, 3H), 3.88 (s, 9H), 3.45 (s, 3H), 0.28 (s, 9H).

\section{1,3,9,11-Tetramethoxy-6-methoxymethyl-14-oxa-benzo[ $\alpha]$ naphthacen-8-one (23).}

A solution of the above crude product $22 \mathrm{c}(42.3 \mathrm{mg})$ and palladium (II) acetate $(17.5 \mathrm{mg}$, $0.078 \mathrm{mmol})$ in anhydrous acetonitrile $(1.32 \mathrm{~mL})$ was refluxed for $12 \mathrm{~h}$. After being allowed to cool to room temperature, the reaction mixture was concentrated in vacuo and the residue was immediately purified by flash column chromatography (silica gel treated with triethylamine $2.5 \% \mathrm{w} / \mathrm{v}$, dichloromethane and then dichloromethane-methanol 97:3) to give $\mathbf{2 3}$ as a blue substance (20.4 $\mathrm{mg}, 56 \%$ for three steps). 
IR (neat) 2926, 2855, 1659, 1613, 1590, $1536 \mathrm{~cm}^{-1}$;

UV $\lambda \max \left(\mathrm{CH}_{3} \mathrm{CN}\right) / \mathrm{nm} 208$ (log $\left.\varepsilon, 5.24\right), 234$ (5.27), 323 (4.91), 610 (4.45);

${ }^{1} \mathrm{H} \mathrm{NMR}\left(\mathrm{CDCl}_{3}, 300 \mathrm{MHz}\right) \delta 8.43(\mathrm{~s}, 1 \mathrm{H}), 7.32(\mathrm{~s}, 1 \mathrm{H}), 6.64(\mathrm{~d}, J=1.9 \mathrm{~Hz}, 1 \mathrm{H}), 6.50(\mathrm{~d}$, $J=2 \mathrm{~Hz}, 1 \mathrm{H}), 6.40(\mathrm{~d}, J=2.3 \mathrm{~Hz}, 1 \mathrm{H}), 6.26(\mathrm{~d}, J=2.2 \mathrm{~Hz}, 1 \mathrm{H}), 6.21(\mathrm{~s}, 1 \mathrm{H}), 4.70(\mathrm{~s}, 2 \mathrm{H})$, $4.00(\mathrm{~s}, 3 \mathrm{H}), 3.94(\mathrm{~s}, 3 \mathrm{H}), 3.88(\mathrm{~s}, 3 \mathrm{H}), 3.88(\mathrm{~s}, 3 \mathrm{H}), 3.49$ (s, 3H);

${ }^{13} \mathrm{C} \mathrm{NMR}\left(\mathrm{CDCl}_{3}, 75.4 \mathrm{MHz}\right) \delta 178.6(\mathrm{C}), 164.3$ (C), 163.9 (C), 161.1 (C), 159.3 (C), 153.5 (C), 150.7 (C), 143.9 (C), 139.3 (C), 133.8 (C), 133.2 (CH), 123.7 (C), $122.2(\mathrm{CH}), 113.4$ (C), $112.2(\mathrm{C}), 109.9(\mathrm{C}), 101.9(\mathrm{CH}), 99.8(\mathrm{CH}), 99.5(\mathrm{CH}), 96.8(\mathrm{CH}), 95.1(\mathrm{CH}), 72.1\left(\mathrm{CH}_{2}\right)$, $58.7\left(\mathrm{CH}_{3}\right), 56.1\left(\mathrm{CH}_{3}\right), 55.9\left(\mathrm{CH}_{3}\right), 55.4\left(\mathrm{CH}_{3}\right), 55.4\left(\mathrm{CH}_{3}\right)$;

MS (DCI, $\mathrm{NH}_{3}+$ isobutane) m/z $499(7), 461\left(\mathrm{MH}^{+}, 100\right)$;

HRMS calcd for $\mathrm{C}_{27} \mathrm{H}_{25} \mathrm{O}_{7}: 461.1600$. Found : $461.1605\left(\mathrm{MH}^{+}\right)$.

\section{Biological Test Protocol}

Supercoiled pKMp27 DNA $(0.5 \mu \mathrm{g})$ was incubated with 6 units of topo I at $37^{\circ} \mathrm{C}$ for 45 minutes in relaxation buffer (50 mM TRIS, pH 7.8, $50 \mathrm{mM} \mathrm{KCL}, 10 \mathrm{mM} \mathrm{MgCl} 2,1 \mathrm{mM}$ Dithiothreitol, 1mM EDTA) in the presence of varying concentrations of the drug under study. Reactions were terminated by adding SDS to $0.25 \%$ and proteins $\mathrm{K}$ to $250 \mu \mathrm{g} / \mathrm{mL}$. DNA samples were then added to the electrophoresis dye mixture $(3 \mu \mathrm{L})$ and electrophoresed in a $1 \%$ agarose gel at room temperature for $3 \mathrm{hrs}$. Gel were stained with ethydium bromide $(1 \mathrm{mg} / \mathrm{mL})$, washed and photographed under UV light. Similar experiments were performed using ethydium-containing agarose gels.

In the presence of topo I inhibitor, the nicked DNA fraction is significantly increased in a dose-dependent manner, reflecting the stabilization of TopoI-DNA complexes. The product $\mathbf{2 3}$ was found active at a dosage of $0.5 \mu \mathrm{g}$. 